\title{
Frequência alimentar no crescimento de alevinos de peixe-rei Odontesthes humensis
}

\author{
Feeding frequency on growth of pejerrey fingerlings \\ Odontesthes humensis
}

\author{
Juvêncio Luís Osório Fernandes Pouey ${ }^{1 *}$; Cleber Bastos Rocha²; Rafael Aldrighi \\ Tavares $^{3}$; Mauro Kaster Portelinha ${ }^{3}$; Sérgio Renato Noguez Piedras ${ }^{4}$
}

\section{Resumo}

\begin{abstract}
A excelente qualidade da carne, a alta taxa reprodutiva e o amplo espectro alimentar sugerem grande potencial para cultivo do peixe-rei. Estudos relacionados à nutrição e alimentação são fundamentais para viabilizar o cultivo de espécies nativas e proporcionar o rápido e eficiente consumo do alimento e reduzir o desperdício de nutrientes. O objetivo deste trabalho foi avaliar a melhor frequência de suprimento de alimento para o crescimento do peixe-rei Odontesthes humensis. Para tal, 600 alevinos com peso médio de $13 \pm 5,9 \mathrm{mg}$ foram distribuídos aleatoriamente em 12 aquários de $50 \mathrm{~L}$. Foram usados quatro tratamentos: ração uma vez/dia, ração três vezes/dia, ração seis vezes/dia e ração oito vezes/dia. Após 32 dias de experimento, foram avaliados: peso médio final, comprimento total, ganho de peso médio, sobrevivência e taxa de crescimento específico. Durante o período experimental a água manteve-se com temperatura de $23 \pm 1,0^{\circ} \mathrm{C}$, oxigênio dissolvido de $6,6 \pm 0,8 \mathrm{mg}$. $\mathrm{L}^{-1}$, pH de 7,5 $\pm 0,4$, a alcalinidade de $45 \pm 5 \mathrm{mg} . \mathrm{L}^{-1} \mathrm{e}$ a amônia total de $0,1 \pm 0,2 \mathrm{mg}$. $\mathrm{L}^{-1}$. As variáveis estudadas apresentaram correlação positiva com a frequência alimentar, porém a alimentação 8 vezes/dia resultou no aumento da heterogeneidade dos animais. Desta forma recomenda-se uma frequência de alimentação de 3 vezes/ dia para alevinos de peixe-rei nesta fase de vida.
\end{abstract}

Palavras-chave: Alimento, dieta, peixe nativo, piscicultura

\begin{abstract}
The excellent quality of pejerrey, high reproductive rate and broad food spectrum suggest great potential for cultivation. Studies related to nutrition and diet are key to facilitate the cultivation of native species and to provide fast and efficient consumption of food and reduce the waste of nutrients. The aim of this work was evaluate the best frequency of food supply Odontesthes humensis growth. For such, 600 fingerlings with average weight of $13 \pm 5.9 \mathrm{mg}$ were equal and randomly distributed in twelve $50 \mathrm{~L}$ experiment tanks. After 32 days of the experiment were evaluated: average final weight, total length, weight gain, specific growth rate and survival. During the experimental period the water temperature was $23 \pm 1.0^{\circ} \mathrm{C}$, oxygen $6.6 \pm 0.8 \mathrm{mg} . \mathrm{L}^{-1}, \mathrm{pH}$ at $7.5 \pm 0.4$, alkalinity at $45 \pm 5 \mathrm{mg} . \mathrm{L}^{-1}$ and total ammonia $0.1 \pm 0.2 \mathrm{mg} . \mathrm{L}^{-1}$. The results of this study indicated a positive correlation between feed frequency and harvest weight, however 8 times/day feed regime resulted in a increase of fish size heterogeneity. Therefore it is recommended that a feed frequency of 3 times/day for pejerrey at this stage of life.
\end{abstract}

Key words: Diet, food, native fish, fish culture

\footnotetext{
${ }^{1}$ D.Sc., Prof. do Laboratório de Ictiologia, Dept ${ }^{\mathrm{o}}$ de Zootecnia, Universidade Federal de Pelotas, UFPel, Pelotas, RS. E-mail: juvencio@ufpel.tche.br

2 D.Sc., Laboratório de Ictiologia, Dept ${ }^{\circ}$ de Zootecnia, UFPel, Pelotas, RS. E-mail: cbr.vet@gmail.com

${ }^{3}$ M.S., Laboratório de Ictiologia, Dept ${ }^{\circ}$ de Zootecnia, UFPel, Pelotas, RS. E-mail: rafaaldrighi@gmail.com; mkportelinha@gmail. com

${ }^{4}$ D.Sc., Prof. do Laboratório de Ictiologia, Dept ${ }^{0}$ de Zootecnia, UFPel, Pelotas, RS. E-mail: sergiopiedras@hotmail.com

* Autor para correspondência
} 


\section{Introdução}

Os peixes-rei de água doce são comuns nas lagoas costeiras do sul do Brasil, Uruguai e Argentina. Na zona sul do Rio Grande do Sul, o registro do gênero Odontesthes, deve-se a Grosser, Koch e Drugg-Hahn (1994) e Bemvenuti (1995), que citam para as lagoas Mirim e Mangueira, as espécies Odontesthes bonariensis, O. humensis e $O$. retropinnis. A excelente qualidade de sua carne, alta taxa reprodutiva e amplo espectro alimentar sugerem grande potencial para cultivo (CASEMIRO; HAHN; RANGEL, 2003; ROS; DOMÁNICO, 2006). Recentemente, devido à busca de alternativas de espécies locais, para substituir a crescente invasão de espécies exóticas usadas na piscicultura regional, o peixe-rei, $O$. bonariensis, tem sido objeto de estudos relacionados a sua reprodução e bioecologia (MIRANDA; SOMOZA, 2001). Entretanto são raros os estudos sobre os aspectos nutricionais, e os existentes são relacionados a $O$. bonariensis (PIEDRAS; POUEY, 2004; MIRANDA et al., 2006), não havendo referências sobre $O$. humensis.

Estudos relacionados à nutrição e alimentação são fundamentais para viabilizar o cultivo de espécies nativas (FRASCA-SCORVO; CARNEIRO; MALHEIROS, 2007). Uma alimentação de forma adequada além de proporcionar melhor desempenho dos animais criados, resulta em ganhos econômicos e evita o lançamento de excedentes de rações no ambiente (CARNEIRO; MIKOS, 2005). Para Türker e Dernekbasi (2006), o processo de alimentação e ingestão em peixes, é resultado da interação de diversos fatores entre o animal (sistema hormonal) e o ambiente (condições ambientais), sendo que o desenvolvimento de técnicas de alimentação tem dois objetivos principais: proporcionar o rápido e eficiente consumo do alimento e reduzir o desperdício de nutrientes.

Da mesma forma que a alimentação representa o maior custo econômico na produção de alevinos, fazse importante com que a frequência alimentar seja um fator a ser considerado nos custos de produção e na qualidade da água dos cultivos intensivos
(DAVIS; INKO-TARIAH; AMACHREE，2006; BOOTH et al., 2008). Volkoff e Peter (2006) afirmam que frequência alimentar é um fator determinante na sobrevivência, no crescimento, na conversão alimentar e na composição corporal do peixe.

Portanto este trabalho teve por objetivo identificar a frequência no fornecimento de alimento, que proporcione melhor crescimento de alevinos de peixe-rei (O. humensis).

\section{Material e Métodos}

Em 12 aquários com capacidade de $50 \mathrm{~L}$, foram distribuídos 600 alevinos de peixe-rei com peso médio de $13 \pm 5,9 \mathrm{mg}$, totalizando 50 animais por aquário. Para obtenção do peso inicial médio, 40 alevinos do mesmo lote formam eutanasiados com aprofundamento anestésico (solução de benzocaína sódica $200 \mathrm{mg} . \mathrm{L}^{-1}$ ) e pesados em balança digital. Os aquários foram instalados em um sistema fechado com capacidade de $2300 \mathrm{~L}$, com controle de temperatura e filtro biológico. A cada seis dias os aquários eram limpos por sifonagem e a água do sistema substituída em 50\%. Para manter o equilíbrio eletrolítico e como medida profilática, foram mantidos com salinidade entre 2 e $3 \%$ adicionando-se cloreto de sódio $(\mathrm{NaCl})$. A qualidade da água foi monitorada três vezes por semana, controlando-se os níveis de oxigênio dissolvido e temperatura (oxímetro digital modelo 55 da YSI), pH (potenciômetro modelo AT 310), condutividade (F-1000, Bernauer Aquicultura), alcalinidade e amônia total, seguindo metodologia sugerida por APHA (1998).

O delineamento experimental utilizado foi completamente ao acaso, com quatro tratamentos em triplicata. Os animais forma alimentados à uma taxa de $100 \%$ da biomassa dia, com ração $\mathrm{INVE}^{\circledR}$ N5/8 com $57 \%$ de proteína bruta e $4160 \mathrm{Kcal} . \mathrm{Kg}^{-1}$ de energia digestível. Os tratamentos foram: ração uma vez/dia, ração três vezes/dia, ração seis vezes/ dia e ração oito vezes/dia. Todos os tratamentos receberam, como primeira alimentação do dia, 
uma porção de nauplios artemia a vontade, como recomendado por Miranda et al. (2006).

Ao final de 32 dias de experimento foram avaliados: peso médio final, comprimento total, ganho de peso médio, taxa de crescimento específico, conforme Legendre e Kerdchuen (1995), através da equação: TCE $=100$ (ln Pm final $-\ln$ Pm inicial) $(\mathrm{t})^{-1}$, em que $\mathrm{ln}=$ logaritmo neperiano; $\mathrm{Pm}$ = peso médio; e $\mathrm{t}=$ tempo em dias e sobrevivência.

Os dados foram submetidos à análise de variância. Quando houve diferença significativa entre os tratamentos, as médias foram comparadas pelo teste de Duncan a 5\% de significância. Todas as análises estatísticas foram realizadas com o auxílio do programa Statistica ${ }^{\circledR}$ 5.0.

\section{Resultados e Discussão}

Durante o período experimental a temperatura da água foi de $23 \pm 1,0{ }^{\circ} \mathrm{C}$, o oxigênio $6,6 \pm 0,8$ mg.L $\mathrm{L}^{-1}$, o pH em 7,5 $\pm 0,4$, a alcalinidade em $45 \pm 5$ mg. $\mathrm{L}^{-1}$ e a amônia total $0,1 \pm 0,2 \mathrm{mg} \cdot \mathrm{L}^{-1}$.

Os animais alimentados 3 vezes/dia apresentaram um peso final, comprimento total e ganho de peso médio superior aos alimentados uma vez/dia, mas não diferiram dos peixes alimentados de $6 \mathrm{e}$ 8 vezes/dia (Tabela 1). Embora existam poucos registros sobre frequência alimentar de peixes na fase de alevinos, os resultados obtidos são similares aos registrados para peixe-rei $O$. argentinensis (SAMPAIO; OLIVEIRA; TESSER, 2007) e para o catfish Hetherobrancus longifilis (DAVIS; INKOTARIAH; AMACHREE, 2006). Dwyer et al. (2002) estudando o linguado Limanda ferruginea concluíram que o melhor desempenho é obtido quando a frequência alimentar é 4 vezes/dia, e que a frequência alimentar de 2 vezes/dia resulta em um aumento significativo do coeficiente de variação do peso. Estes autores sugerem que a variação no crescimento de animais alimentados em diferentes frequências pode ser resultante da competição entre os animais e do estabelecimento de hierarquia no grupo. Vários estudos (SANCHEZ-VASQUEZ; TABATA, 1998; CHEN et al., 2007) demonstram que o ritmo circadiano afeta a intensidade com que os peixes procuram o alimento, de maneira que estudos neste sentido poderão fornecer informações mais precisas sobre a frequência alimentar ideal para cada espécie e fase de vida dos peixes cultivados.

Tabela 1. Peso inicial (PI), peso final (PF), comprimento total (CT), ganho de peso (GP), taxa de crescimento específico (TCE) e sobrevivência (Sob.) média e desvio padrão dos alevinos de peixe-rei Odontesthes humensis submetidos a diferentes frequências alimentares.

\begin{tabular}{lrrrr}
\hline \multirow{2}{*}{ Variáveis } & \multicolumn{4}{c}{ Frequência alimentar } \\
\cline { 2 - 5 } & \multicolumn{1}{c}{ 1x/dia } & \multicolumn{1}{c}{ 3x/dia } & \multicolumn{1}{c}{8 x/dia } \\
\hline PI (mg) & $13,0 \pm 5,9$ & $13,0 \pm 5,9$ & $13,0 \pm 5,9$ & $13,0 \pm 5,9$ \\
PF (mg) & $126,7 \pm 8,1^{\mathrm{b}}$ & $161,7 \pm 9,6^{\mathrm{a}}$ & $167,0 \pm 13,0^{\mathrm{a}}$ & $178 \pm 38,2^{\mathrm{a}}$ \\
CT (mm) & $26,5 \pm 0,7^{\mathrm{b}}$ & $28,3 \pm 0,7^{\mathrm{a}}$ & $28,4 \pm 0,7^{\mathrm{a}}$ & $29,0 \pm 1,8^{\mathrm{a}}$ \\
GP (mg) & $113,67 \pm 8,1^{\mathrm{b}}$ & $148,7 \pm 9,6^{\mathrm{a}}$ & $154,3 \pm 11,8^{\mathrm{a}}$ & $165,3 \pm 38,9^{\mathrm{a}}$ \\
TCE (\%) & $11,8 \pm 0,6^{\mathrm{c}}$ & $14,7 \pm 0,1^{\mathrm{a}}$ & $14,3 \pm 0,2^{\mathrm{b}}$ & $15,1 \pm 0,2^{\mathrm{a}}$ \\
Sob. (\%) & $44,0 \pm 1,6^{\mathrm{b}}$ & $71,3 \pm 9,9^{\mathrm{a}}$ & $78,7 \pm 9,8^{\mathrm{a}}$ & $69,3 \pm 4,6^{\mathrm{a}}$ \\
\hline
\end{tabular}

*Letras minúsculas diferentes, na mesma linha, indicam diferença significativa $(\mathrm{p} \leq 0,05)$ pelo teste de Duncan.

Fonte: Elaboração dos autores. 
Em relação à taxa de crescimento específico, observou-se uma redução quando a frequência alimentar foi de 6 vezes/dia. Carneiro e Mikos (2005) observaram que o crescimento de alevinos de jundiá (Rhamdia quelen) submetidos à frequência alimentar (1 a 4 vezes/dia) não foi afetado, mas sugerem que uma maior frequência alimentar possibilita um maior controle do cultivo. Em contrapartida, Canton et al. (2007) afirmam que juvenis de jundiá, com peso médio de $8,0 \mathrm{~g}$ têm melhor desempenho quando alimentados no mínimo 4 vezes/dia. Esta discrepância de resultados sugere que outras variáveis, como condições ambientais e condições experimentais podem afetar o desempenho dos animais. Por outro lado, existe um consenso entre diversos autores, de que, na fase de alevinos, uma maior frequência alimentar proporciona melhor desempenho dos peixes. Este fator pode ser justificado pelo fato dos peixes nas fases jovens apresentarem metabolismo elevado (SCHNAITTACHER; KING; BERLINSKY, 2005).

Os peixes alimentados 8 vezes/dia tiveram um aumento significativo do desvio padrão das variáveis peso final, comprimento total, ganho de peso médio e taxa de crescimento específico, resultando em animais heterogêneos, o que não é desejável. $\mathrm{O}$ aumento da heterogeneidade dos animais pode ser atribuído ao estabelecimento de uma hierarquia, em que os animais maiores se alimentam primeiro, diminuindo a quantidade de alimento disponível para os demais peixes, já que a quantidade diária de alimento fornecido foi a mesma em todos os tratamentos. De acordo com Dias Júnior e Mourgués-Schurter (2001) uma das formas de evitar os problemas causados pela hierarquia é aumentar o tempo de disponibilidade do alimento. De acordo com Henken, Kleingeld e Tijssen (1985) o consumo de alimento em curtos intervalos de tempo diminui a eficiência digestiva, podendo afetar o desempenho e homogeneidade dos animais criados. Sampaio, Oliveira e Tesser (2007) não encontraram variação significativa no coeficiente de variação do peso em O. argentinensis, alimentados 4 vezes/dia.
De acordo com Boujard e Leattherlad (1992), fatores como horários e tempo disponível para alimentação, têm relação direta com mecanismos fisiológicos que controlam a liberação de enzimas e hormônios, que por sua vez afetam a frequência alimentar das diferentes espécies de peixes.

Os resultados mostraram uma baixa sobrevivência (44\%) nos animais alimentados uma vez/dia, nos demais tratamentos a sobrevivência foi aproximadamente de $70 \%$. Resultados semelhantes foram observados por Miranda et al. (2006) onde a sobrevivência de $O$. bonariensis em tanques de terra alimentados com artemia, zooplancton e ração artificial (2 e 4 vezes/dia) foi de $73 \%$. Já Sampaio, Oliveira e Tesser (2007) constataram uma sobrevivência de $90 \%$ de alevinos de $O$. argentinensis alimentados com ração, entre 1 e 4 vezes/dia.

\section{Conclusões}

Os resultados obtidos neste trabalho sugerem que alevinos de $O$. humensis durante os primeiros 30 dias de vida devem ser alimentados 3 vezes/dia, a alimentação 8 vezes/dia resulta em aumento da heterogeneidade dos animais

\section{Referências}

AMERICAN PUBLIC HEALTH ASSOCIATION APHA. Standard methods for the examination of water and wastewater. Washington: American Public Health Association, 1998. $937 \mathrm{p}$.

BEMVENUTI, M. A. Odontesthes mirinensis, sp.n. um novo peixe-rei (Pisces, Atherinidae, Atherinopsinae) para o extremo Sul do Brasil. Revista Brasileirade Zoologia, Curitiba, v. 12, n. 4, p. 881-903, 1995.

BOOTH, M. A.; TUCKER, B. J.; ALLAN, G. L.; STEWART FIELDER, D. Effect of feeding regime and fish size on weight gain, feed intake and gastric evacuation in juvenile Australian snapper Pagrus auratus. Aquaculture, Amsterdãn, v. 282, n. 1-4, p. 104110, 2008.

BOUJARD, T.; LEATHERLAND, J. F. Circadian rhythms and feeding time in fishes. Environmental Biology of Fishes, Corvallis, v. 35, n. 2, p. 109-131, 1992. 
CANTON, R.; WIENGARTNER, M.; FRACALOSSI, D. M.; ZANIBONI-FILHO, E. Influência da frequência alimentar no desempenho de juvenis de jundiá. Revista Brasileira de Zootecnia, Viçosa, v. 36, n. 4, p. 749-753, 2007.

CARNEIRO, P. C. F.; MIKOS, J. D. Frequência alimentar e crescimento de alevinos de jundiá Rhamdia quelen. Ciência Rural, Santa Maria, v. 35, n. 1, p. 187-191, 2005.

CASEMIRO, F. A. S.; HAHN, N. S.; RANGEL, T. F. L. V. B. Diet and trophic ecomorphology of the Odontesthes bonariensis, of the Salto Caxias reservoir, rio Iguaçu, Paraná, Brazil. Neotropcal Ichthyoloy, Porto Alegre, v. 1, n. 2, p. 127-134, 2003.

CHEN, W. M.; UMEDA, N.; MITSUBOSHI, T.; HIRAZAWA, N. Circadian self-feeding rhythms in greater amberjack Seriola dumerli (Risso). Journal of Fish Biology, Liverpool, v. 70, n. 2, p. 451-461, 2007.

DAVIS, O. A.; INKO-TARIAH, M. B.; AMACHREE, D. Growth response and survival of Heterobranchus longifilis fingerlings fed at different feeding frequencies. African Journal of Biotechnology, Nairobi, v. 5, n. 9, p. 778-780, 2006.

DIAS JÚNIOR, W.; MOURGUÉS-SCHURTER, L. R. Comportamento alimentar, determinação do horário de fornecimento e do tempo de disponibilidade da ração para Leporinus obtusidens VALENCIENNES, 1847 (Osteichthyes, Characiformes, Anastomidae) (Piau). Ciência e Agrotecnologia, Lavras, v. 5, n. 25, p. 10431050, 2001.

DWYER, K. S.; BROWN, J. A.; PARRISH, C.; LALL, S. P. Feeding frequency affects food consumption, feeding pattern and of juvenile yellowtail flounder (Limanda ferruginea). Aquaculture, Amsterdãn, v. 213, n. 1, p. 279292, 2002.

FRASCA-SCORVO, C. M.; CARNEIRO, D. J.; MALHEIROS, E. B. Efeito do manejo alimentar no desempenho do matrinxã Brycon amazonicus em tanques de cultivo. Acta Amazônica, Manaus, v. 37, n. 4, p. 621628, 2007.

GROSSER, K. M.; KOCH, W. R.; DRUGG-HAHN, S. Ocorrência e distribuição de peixes na Estação Ecológica do Taim, Rio Grande do Sul, Brasil (Pisces, Teleostomi). Iheringia: SérieZoologia, Porto Alegre, v. 77, n. 1, p. 8998, 1994.

HENKEN, A. M.; KLEINGELD, D. W.; TIJSSEN, P. A. $\mathrm{T}$. The effect or feeding level on apparent digestibility of dietary dry mater, crude protein and gross energy in the African catfish (Claris gariepinus). Aquaculture, Amsterdãn, v. 51, n. 1, p. 1-11, 1985.
LEGENDRE, M.; KERDCHUEN, N. Larval rearing of an African Catfish Heterobranchus longifilis (Teleostei, Claridae): effect of dietary lipids on growth surviral and fatty acid composition of fry. Aquatic Living Resources, Cambridge, v. 8, n. 4, p. 355-363, 1995.

MIRANDA, L. A.; BERSAIN, G. E.; VELASCO, C. A. M.; SHIROJO, Y.; SOMOZA, G. M. Natural spawning and intensive culture of pejerrey Odontesthes bonariensis juveniles. Biocell, Mendoza, v. 30, n. 1, p. 157-162, 2006.

MIRANDA, L. A.; SOMOZA, G. M. Biologia reprodutiva del pejerrey Odontesthes bonariensis: Diferenciacion sexual y endocrinología de la reproducción. Aspectos básicos y su potencial aplicación en la acuicultura. In: GROSMAN, F. Fundamentos biológico, econômicos y sociales para correcta gestion del recurso pejerrey. Buenos Aires: Editora Astianax, 2001. p. 41-45.

PIEDRAS, S. R. N.; POUEY, J. L. O. F. Alimentação de alevinos de peixe-rei (Odonthestes bonariensis) com dietas naturais e artificiais. Ciência Rural, Santa Maria, v. 34, n. 4, p. 1203-1206, 2004.

ROS, A. E.; DOMÁNICO, A. Growth of argentine silverside (Odontesthes bonariensis) stocked at low densities in two oligohaline shallow lakes (Buenos Aires Province, Argentina). Revista de Investigaciones e Desarrolo Pesquero, Mar del Plata, v. 1, n. 18, p. 75-80, 2006.

SAMPAIO, L. A.; OLIVEIRA, M.; TESSER, M. B. Produção de larvas e juvenis de peixe - rei Odonteshtes argentinensis submetidos a diferentes frequências alimentares. Revista Brasileira de Agrociência, Pelotas, v. 13, n. 2, p. 271-274, 2007.

SANCHEZ-VAZQUEZ, F. J.; TABATA, M. Circadian thythms of demand-feeding and locomotor actitvity in rainbow trout. Journal of Fish Biology, Liverpool, v. 52, n. 2, p. 255-267, 1998.

SCHNAITTACHER, G.; KING, V. W.; BERLINSKY, D. L. The effects of feeding frequency on growth of juvenile Atlantic halibut, Hippoglossus hippoglossus L. Aquaculture Research, Oxford, v. 36, n. 4, p. 370-377, 2005.

TÜRKER, A.; DERNEKBASI, S. Y. Effects of restricted feeding on performances of rainbow trout (Oncorhynhus mykiss). Journal of Faculty of Agriculture, Omü, v. 21, n. 2, p. 190-194, 2006.

VOLKOFF, H.; PETER, R. E. Feeding behavior of fish and its control. Zebrafish, New Rochelle, v. 3, n. 2, p. 131-137, 2006. 
